\title{
Tyrosinase Inhibitory Effects of Sargachromanol G, Sargachromanol I and Mojabanchromanol b isolated from Myagropsis myagroides
}

\author{
K. B. W. R. KIM, S. M. JEONG, M. J. KIM ${ }^{1}$ AND D. H. AHN ${ }^{1 *}$ \\ Institute of Fisheries Sciences, Pukyong National University, Busan 46041, ${ }^{1}$ Department of Food Science and Technology/ \\ Institute of Food Science, Pukyong National University, Busan 48513, Republic of Korea
}

Kim et al.: Tyrosinase Inhibitory Effects of Chromenes from Myagropsis myagroides

\begin{abstract}
The aim of this study was to elucidate the effect of Myagropsis myagroides extract on tyrosinase activity. Inhibition of tyrosinase was observed in the presence of an $n$-hexane fraction of Myagropsis myagroides. Separation of the $n$-hexane fraction using silica gel column chromatography yielded the chloroform:methanol (50:1) fraction, which showed the highest tyrosinase inhibitory activity. After several separation and purification steps, sargachromanol G, sargachromanol I, and mojabanchromanol b were obtained. These 3 chromene compounds displayed strong tyrosinase inhibitory activity. It could be concluded that Myagropsis myagroides is a potential source of natural antimelagenic compounds.
\end{abstract}

Key words: Mojabanchromanol b, Myagropsis myagroides, sargachromanol G, sargachromanol I, Tyrosinase inhibitory effect

Melanogenesis is the biosynthetic pathway for the formation of the pigment melanin in skin melanocyte. Melanocytes produce two types of melanin: pheomelanin and eumelanin formed by conjugation of glutathione or cysteine. Melanogenesis is initiated with the oxidation of L-tyrosine to L-dopaquinone, the precursor of pheomelanin and eumelanin, by tyrosinase ${ }^{[1]}$; this enzyme is therefore important for mammalian melanin synthesis in melanocytes. Tyrosinase is a multifunctional membrane-bound type 3 copper-containing glycoprotein, and is expressed only in melanocytes. Melanogenesis can be inhibited by inhibition of tyrosinase or by inhibition of melanocyte

*Address for correspondence

E-mail: dhahn@pknu.ac.kr

January-February 2020 metabolism ${ }^{[2]}$. Physiologically, activation of tyrosinase in skin stimulates melanin production to protect skin damaged by UVA and UVB radiation ${ }^{[3,4]}$; however, the accumulation of melanin can cause hyperpigmentation of skin and dermatological disorders such as freckles and age spots. Tyrosinase inhibition reduces transformation of L-tyrosine to melanin, slowing and decreasing skin hyperpigmentation ${ }^{[5]}$.

This is an open access article distributed under the terms of the Creative Commons Attribution-NonCommercial-ShareAlike 3.0 License, which allows others to remix, tweak, and build upon the work non-commercially, as long as the author is credited and the new creations are licensed under the identical terms

Accepted 18 December 2019 Revised 12 October 2019 Received 23 August 2019 Indian J Pharm Sci 2020;82(1):170-173

January-February 2020 
Myagropsis myagroides is a brown algae that inhabits the coasts of South Korea, China, and Japan. There are some reports showing that M. myagroides has antibacterial $^{[6]}$, antidiabetic ${ }^{[7]}$, hepatopotective ${ }^{[8]}$ and antiinflammatory ${ }^{[9-12]}$ properties due to the presence of pigments and polyphenols. On the other hand, there is no research on the inhibition of melanogenesis by extract of M. myagroides. In the present study, evidence was provided that tyrosinase activity is inhibited by extracts of $M$. myagroides, supporting the view that $M$. myagroides contains antimelanogenic compounds.

M. myagroides was harvested from Song-Jeong, Busan, Korea in March 2011. M. myagroides was washed 3 times with tap water to remove salt, epiphytes, sand and then dried naturally. The dried M. myagroides was lyophilized and pulverized. The powder was stored at $-20^{\circ}$. A voucher specimen (MBRB0078) has been deposited at the Marine Brown Algae Resources Bank.

Powdered M. myagroides $(1.5 \mathrm{~kg}$ ) was extracted with methanol $(\mathrm{MeOH})$ for $24 \mathrm{~h}$ at room temperature with an agitator (H-8020, Dongwon Science Co., Busan, Korea) and filtered via vacuum suction with a Büchner funnel. The filtrate was evaporated in vacuo to obtain $\mathrm{MeOH}$ extract, which were suspended in distilled $\mathrm{H}_{2} \mathrm{O}$ and then successively partitioned with $n$-hexane $(13 \mathrm{~g}), \mathrm{CHCl}_{3}(11 \mathrm{~g})$, ethyl acetate (EtOAc, $\left.0.5 \mathrm{~g}\right)$ and $n$-BuOH (butanol, $4.4 \mathrm{~g}$ ), respectively. The $n$-hexane soluble fraction $(10 \mathrm{~g})$ was chromatographed on a silica gel column and eluted with $\mathrm{CHCl}_{3}-\mathrm{MeOH}(\mathrm{CM}$, 100:0 1:1) to yield 6 fractions. The CM (50:1) fraction (1.6 g) was subjected to a Sephadex LH-20 column chromatography and ODS Sepak cartridge (60 90\% methanol) to 7 fractions. Of these fractions, the fraction 4 (242 mg) was further purified by Sephadex LH-20 column chromatography and DOS Sepak cartridge (50 60\% methanol). The fractions were separated using ODS high-performance liquid chromatographyphotodiode array detection (HPLC-PDA; Hitachi, Chatsworth, CA, USA) and found to contain compounds such as sargachromnaol G (conditions$70 \%$ methanol, $9 \mathrm{ml} / \mathrm{min}$ ), sargachromanol I (conditions$70 \%$ methanol, $9 \mathrm{ml} / \mathrm{min}$ ), and mojabanchromanol b (conditions- $75 \%$ methanol, $3 \mathrm{ml} / \mathrm{min}$ ), which were isolated with yields of 20,52 and $2 \mathrm{mg}$, respectively. The UV spectra of eluted compounds were recorded from 200 to $500 \mathrm{~nm}$. Their structures were elucidated by ${ }^{1} \mathrm{H}$ NMR (600 MHz, $\mathrm{CDCl}_{3}$; JNM-ECA600, Jeol Ltd, Tokyo, Japan) and ${ }^{13} \mathrm{C}$ NMR $\left(150 \mathrm{MHz}, \mathrm{CDCl}_{3}\right)$, ${ }^{1} \mathrm{H}-{ }^{1} \mathrm{H}$ COSY, HMQC, HMBC, and EI-MS (JMS-700, Jeol Ltd) spectra.
Tyrosinase inhibition assays were performed using mushroom tyrosinase (Sigma-Aldrich Co., St. Louis, MO, USA) as described previously, with some modification $^{[13]}$. Briefly, $M$. myagroides fraction $(10 \mu \mathrm{l})$ was added to a reaction mixture containing 1500 unit $/ \mathrm{ml}$ mushroom tyrosinase $(10 \mu \mathrm{l}), 0.1 \mathrm{M}$ sodium phosphate buffer $(\mathrm{pH} 6.5,110 \mu \mathrm{l})$ and $4 \mathrm{mM}$ L-tyrosine ( $20 \mu$ l, Sigma-Aldrich Co.) in a 96-well plate for $20 \mathrm{~min}$ at $37^{\circ}$. Tyrosinase activity was measured by monitoring the formation of L-DOPAchrome, indicated by an increase in absorbance at $490 \mathrm{~nm}$ using UV/Vis spectrophotometer.

Data was expressed as mean \pm standard error of the mean. Statistical evaluation was carried out using analysis of variance (ANOVA) in SAS software (SAS Institute, Inc., Cary, NC, USA) and the differences between means were assessed using Duncan's multiple range test at $\mathrm{p}<0.05$.

Tyrosinase is an enzyme containing copper at the active site and acts on the initial reaction of melanogenesis ${ }^{[1]}$. To investigate effect of the tyrosinase inhibitory activity, the amount of DOPA chrome was determined. The tyrosinase inhibitory activity of the $n$-hexane fraction of the methanol extract was $25.64 \%$ at $5 \mathrm{mg} /$ $\mathrm{ml}$ (Table 1), higher than that of the other fractions. Fraction CM (50:1) was obtained by silica gel column chromatography, which showed the highest inhibition activity of $36.46 \pm 4.65 \%$ at $5 \mathrm{mg} / \mathrm{ml}$. Seven fractions were obtained from fraction CM (50:1) using a Sephadex LH-20 column chromatography and ODS separk cartridge, among which fractions 3 and 4 inhibited tyrosinase $13.07 \pm 1.23$ and $38.89 \pm 5.70 \%$, respectively (Table 1). Similar levels of tyrosinase inhibition by seaweed extracts have been reported previously. For example, it was reported that tyrosinase activity is partially inhibited by methanol extracts of Sargassum siliquastrum and Ecklonia cava ${ }^{[14]}$. In particular, the methanol extract of $S$. siliquastrum had an inhibitory

TABLE 1: TYROSINASE INHIBITORY ACTIVITY OF SUB-FRACTIONS FROM n-HEXANE FRACTION OF MYAGROPSIS MYAGROIDES

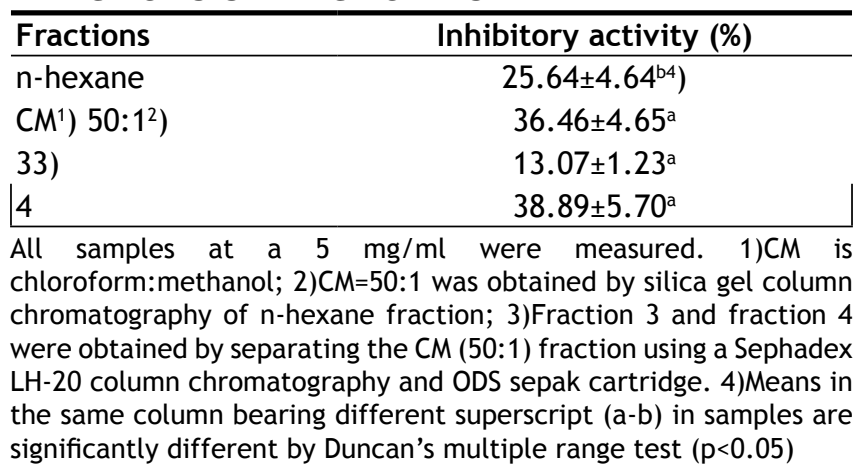


A)

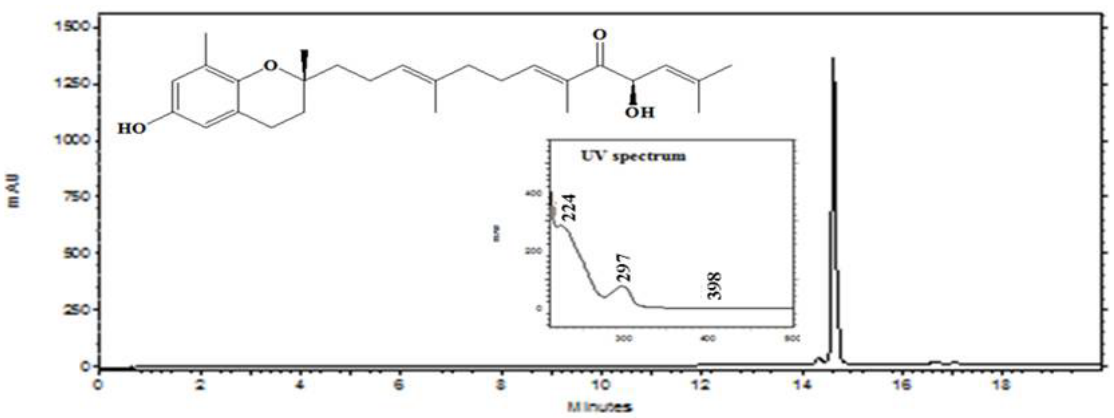

B)

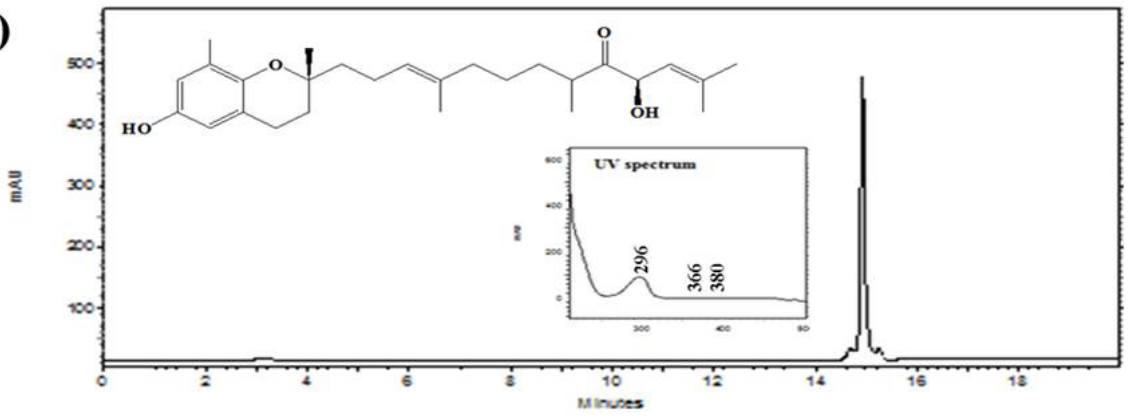

C)

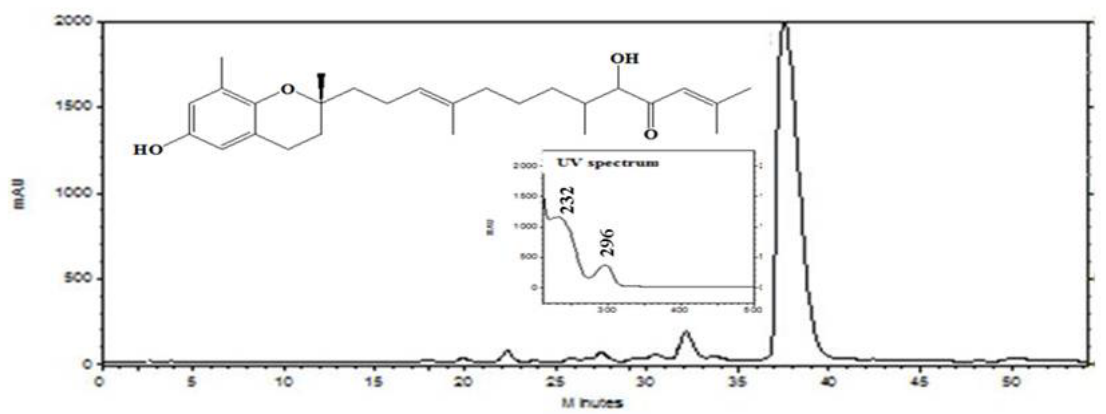

Fig. 1: HPLC chromatograms and UV spectra of constituents from Myagropsis myagroides methanol extract HPLC profiles and UV spectra of (A) Sargachromanol G, (B) Sargachromanol I, and (C) Mojabanchromanol b isolated from Myagropsis myagroides methanol extract

TABLE 2: TYROSINASE INHIBITORY ACTIVITY OF THE 3 CHROME COMPOUNDS ISOLATED FROM FRACTION 4 OF MYAGROPSIS MYAGROIDES

Isolated compounds

Sargachromanol G

Sargachromanol I

Mojabanchromanol b

Arbutin Inhibitory activity (\%)

$\left.41.27 \pm 4.70^{\mathrm{b} 1}\right)$

$19.92 \pm 8.59^{c}$

$48.58 \pm 2.42^{\mathrm{b}}$

$74.47 \pm 1.17^{\mathrm{a}}$

All samples at a $5 \mathrm{mg} / \mathrm{ml}$ were measured. 1)Means in the same column bearing different superscripts $(a-b)$ are significantly different by Duncan's multiple range test $(p<0.05)$

activity of $50 \%$. In addition, ethyl acetate extracts of Endarachne binghamiae ${ }^{[15]}$ and Undaria pinnatifida ${ }^{[5]}$ has shown by measurement of activity of intracellular tyrosinase in mouse melanocytes or melanoma cells to exhibit a high inhibition rate of melanin synthesis. However, the melanin synthesis inhibitory compounds in seaweed extracts have not been analyzed.

Fraction 4, which exhibited high inhibitory activity, was purified by HPLC and 3 chromene compounds were isolated, which were sargachromanol $\mathrm{G}\left(\mathrm{C}_{27} \mathrm{H}_{38} \mathrm{O}_{4}\right)$, sargachromanol $\mathrm{I}\left(\mathrm{C}_{27} \mathrm{H}_{40} \mathrm{O}_{4}\right)$, and mojabanchromanol b $\left(\mathrm{C}_{27} \mathrm{H}_{40} \mathrm{O}_{4}\right)$ (fig. 1). Tyrosinase inhibitory activity was detected with all 3 compounds and mojabanchromanol b exhibited the highest inhibitory activity (48.58 $\pm 2.42 \%)$, followed by sargachromanol G (41.27 $\pm 4.70 \%)$ and sargachromanol I (19.92 $\pm 8.59 \%$, Table 2). These 3 compounds have already been reported ${ }^{[16,17]}$, but theinhibitoryactivityoftyrosinaseisnot reported. Sargachromanol G and I have antiinflammatory and antioxidant effects, which are found in the brown algae family. Sargachromanol $G$, isolated from $S$. siliquastrum, was reported to inhibit collagenase activity and exhibited antiinflammatory effect in osteoblasts $^{[18]}$, while sargachromanol I showed antioxidant potency ${ }^{[19]}$ and $\alpha$-amylase inhibitory activity $^{[7]}$. Sargachromnenol $G$ was also been isolated from $S$. horneri and in this case, it was reported to suppress UVA-induced photoaging ${ }^{[20]}$. Mojabanchromanol b was shown to have an antiinflammatory effect by 
suppressing the release of cytokines and nitric oxide ${ }^{[9]}$. Thus, these compounds are known to exert various biological effects. However, further experiments on these 3 compounds, including in vivo experiments, are needed to investigate their mechanisms.

In summary, this study demonstrated for the first time that sargachromanol G, sargachromanol I and mojabanchromanol b compounds isolated from M. myagroides exhibited tyrosinase inhibitory activity. In particular, it was found that mojabanchromanol b had greater effect on tyrosinase inhibition than other compounds. This finding suggested that compounds extracted from $M$. myagroides, especially mojabanchromanol $\mathrm{b}$, could have applications in the food industry, medicine and as a natural skin-whitening agent.

\section{Acknowledgements:}

This research was supported by the Basic Research Program through the National Research Foundation of Korea (NRF) funded by the Ministry of Education (No. 2012R1A6A1028677).

\section{REFERENCES}

1. Pillaiyar T, Manickam M, Namasivayam V. Skin whitening agents: medicinal chemistry perspective of tyrosinase inhibitors. J Enzyme Inhib Med Chem 2017;32:403-425.

2. D'Mello SA, Finlay GJ, Baguley BC, Askarian-Amiri ME. Signaling pathways in melanogenesis. Int $\mathrm{J}$ Mol Sci 2016;17:1144-62.

3. Gilchrest BA, Eller MS. DNA photodamage stimulates melanogenesis and other photoprotective responses. J Investig Dermatol Symp Proc 1999;4:35-40.

4. Gilchrest BA, Eller MS, Geller AC, Yaar M. The pathogenesis of melanoma induced by ultraviolet radiation. N Engl J Med 1999;340:1341-8.

5. Kim MJ, Kim DS, Yoon HS, Lee WJ, Lee NH, Hyun CG. Melanogenesis inhibitory activity of Korean Undaria pinnatifida in mouse B16 melanoma cells. Interdiscip Toxicol 2014;7:89-92.

6. Lee SY, Kim KBWR, Lim SI, Ahn DH. Antibacterial mechanism of Myagropsis myagroides extract on Listeria monocytogenes. Food Control 2014;42:23-28.

7. Pak WM, Kim KB, Kim MJ, Cho JY, Ahn DH. Inhibitory effect of hexane fraction from Myagropsis myagroides on pancreatic $\alpha$-amylase in vitro. J Microbiol Biotechnol 2015;25:328-333.
8. Wong CK, Ooi VEC, Ang PO. Hepatoprotective effect of seaweeds' methanol against carbon tetrachloride-induced poisoning in rats. Hydrobiologia 2004;512:267-270.

9. Jeong DH, Kim KBWR, Kim MJ, Kang BK, Ahn DH. Antiinflammatory activity of methanol extract and n-hexane fraction mojabanchromanol b from Myagropsis myagroides. Life Sci 2014;26:12-19.

10. Joung EJ, Lee MS, Choi JW, Kim JS, Shin T, Jung BM, et al. Anti-inflammatory effect of ethanolic extract from Myagropsis myagroides on murine macrophages and mouse ear edema. BMC Complement Altern Med 2012;12:1-11.

11. Kim S, Kim JI, Choi JW, Kim M, Yoon NY, Choi CG, et al. Anti-inflammatory effect of hexane fraction from Myagropsis myagroides ethanolic extract in lipopolysaccharide-stimulated BV-2 microglial cells. J Pharm Pharmacol 2013;65:895-906.

12. Kim S, Lee MS, Lee B, Gwon WG, Joung EJ, Yoon NY, et al. Anti-inflammatory effects of sargachromenol-rich ethanolic extract of Myagropsis myagroides on lipopolysaccharidestimulated BV-2 cells. BMC Complement Altern Med 2014;14:1-14.

13. Kim YM, Yun J, Lee CK, Lee H, Min KR, Kim Y. Oxyresveratrol and hydroxystilbene compounds. Inhibitory effect on tyrosinase and mechanism of action. J Biol Chem 2002;277:16340-16344.

14. Lee HJ, Kim JH, Lee CH, Kim JS, Kwak ST, Lee KB, et al. Inhibitory activities of seaweeds on prolyl endopeptidase, tyrosinase and coagulation. Kor J Paharmacogn 1999;30:231237.

15. Jeon YS, Jung YJ, Younm JK, Kim YK, Kim SN. Inhibitory effect of Endarachne binghamiae extract on melanin synthesis. Korea J Plant Res 2013;26:526-532.

16. Gwon WG, Joung EJ, Kwon MS, Lim SJ, Utsuki T, Kim HR. Sargachromenol protects against vascular inflammation by preventing TNF- $\alpha$-induced monocyte adhesion to primary endothelial cells via inhibition of $\mathrm{NF}-\mathrm{\kappa B}$ activation. Int Immunopharmacol 2017;42:81-89.

17. Heo SJ, Jang J, Ye BR, Kim MS, Yoon WJ, Oh C, et al. Chromene suppresses the activation of inflammatory mediators in lipopolysaccharide-stimulated RAW 264.7 cells. Food Chem Toxicol 2014;67:169-175.

18. Yoon WJ, Kim KN, Heo SJ, Han SC, Kim J, Ko YJ, et al. Sargachromanol G inhibits osteoclastogenesis by suppressing the activation NF- $\kappa \mathrm{B}$ and MAPKs in RANKL-induced RAW 264.7 cells. Biochem Biophys Res Commun 2013;17:892-897.

19. Kim JA, Kong CS, Kim SK. Effect of Sargassum thunbergii on ROS mediated oxidative damage and identification of polyunsaturated fatty acid components. Food Chem Toxicol 2010;48:1243-9.

20. Kim JA, Ahn BN, Kong CS, Kim SK. Protective effect of chromene isolated from Sargassum horneri against UV-Ainduced damage in skin dermal fibroblasts. Exp Dermatol 2012;21:630-1. 